\title{
Frontiers in modeling and design of bio-inspired armors
}

\author{
Stefano Signetti ${ }^{1}$ and Nicola Maria Pugno ${ }^{1,2,3}$ * \\ ${ }^{1}$ Laboratory of Bio-Inspired and Graphene Nanomechanics, Department of Civil, Environmental and Mechanical Engineering, University of Trento, Trento, Italy \\ ${ }^{2}$ Centre for Materials and Microsystems, Fondazione Bruno Kessler, Povo (Trento), Italy \\ ${ }^{3}$ School of Engineering and Materials Science, Queen Mary University of London, London, UK \\ ${ }^{*}$ Correspondence: nicola.pugno@unitn.it \\ Edited by: \\ Giuseppe Saccomandi, Università di Perugia, Italy \\ Reviewed by: \\ Fernando Fraternali, University of Salerno, Italy
}

Keywords: bio-inspired armors, impact testing, numerical simulation, theoretical modeling, optimization, toughness

Impact behavior of materials and structures is of crucial interest. Indeed, every solid may experience collisions in its "mechanical" life. The topic involves a wide range of engineering applications, even in our everyday life: sports protectors [e.g., helmets, Milne et al. (2014)], sensitive portable electronics (Tempelman et al., 2012), bulletproof body armors (Cuniff, 1999), protection systems for buildings and machineries in the civil or defense sectors (NIST, 2005), improvement of crashworthiness in automotive (Schweizerhof et al., 1992), protection of spacecraft and satellite structures from high-velocity micrometeorite or orbital debris impact (NASA, 2015) are some of the most representative.

Parallel to the primary requirement of an effective protection, straightforwardly achievable with a massive armor, research efforts are aimed at weight saving due to essential and binding needs, such us better ergonomics and flexibility (body armors), transportability (vehicles), and in general a more judicious use of materials. Thus, the real goal is the high specific toughness. For some decades, the answer to these tasks has been the adoption of multilayer of textile and composite materials, (Abrate, 1998; Hoog, 2006) based on synthetic fibers (e.g., Kevlar ${ }^{\circledR}$, Dyneema $^{\circledR}$ ) that have allowed to reach protection levels previously unimaginable with metallic targets. Nowadays, in the era of nanomaterials, we are raising the bar to atomistic 2D materials, like graphene, coupling high resistance (Lee et al., 2008) and flaw tolerance (Zhang et al., 2012) at the nanoscale, even for possible application to nanoarmours (Pugno et al., 2007; Lee et al., 2012, 2014). Alternatively, the same goal may be pursued through smart structural solutions to be employed even with traditional materials, with all the benefits that this option implies. Nature, having worked over the ages for optimizing defense mechanisms against predators attacks or shock loads, is one of the most inspiring sources: as most remarkable examples we mention the coupled hard-soft layers in the Arapaima gigas fish's dermis (Yang et al., 2014), the internal undulated walls of the Bombardier Beetle's (Carabidae, Brachinus) explosion chamber (Lai and Ortiz, 2010), the cross-scale toughening mechanisms in the foam-like structure of dropping fruits (Thielen et al., 2013), dermal armors with scales (Ghosh et al., 2014), and the extreme robustness provided by the spider silk constitutive law (Cranford et al., 2012). On the other side, we could be interested in gaining an efficient strike, like the deadly underwater punch mechanism of the mantis-shrimp (Patek et al., 2005).

Upon impact, several complex physical phenomena take place: elastic-plastic deformation and wave propagation, fracture and fragmentation, heat generation (by yielding and friction), changing of material properties due to strain-rate effects up to phase change. Their occurrence and magnitude depend on the impact velocity that may be very low or up to extreme values ( $>3 \mathrm{~km} / \mathrm{s}$ for hypervelocity impact), with increasing challenges for armor resistance as well as for its accurate modeling. The theoretical description of the basic aspects of impact mechanics (Stronge, 2000; Goldsmith, 1999, 2001) has reached a level of advanced maturity but it is in a sort of stalemate due to the severe mathematical complexity in representing the above mentioned phenomena, which also mutually interact. With high speed calculators and the development of computational methods (e.g., finite element method, FEM), simulation has become the favorite design tool, allowing optimization studies. Nonetheless, the advent of nanomaterials and bio-inspiration is further questioning the capabilities of these tools and pushing modeling research.

The traditional stand-alone experimental approach for armor design according to the philosophy "add material until it stops" it is not viable any more. First, multilayer panels can show crosscurrent behavior in relation to material coupling and interface strength, being even non-optimized for increasing areal density (Signetti and Pugno, 2014, Figure 1). Technological and economic limits in large scale production of nanomaterials, the difficulties in their manipulation or in their structural arrangement into complex bio-inspired structures require a systematic and reliable design process able to provide a tentative target optimum. With mere experiments is nearly impossible to investigate the whole design space for understanding still unexplained mechanism in order to mimic nature and, why not, do even better.

Going down to nanoarmours at atomic scale, we enter in a new world with completely unexplored scenarios. Deformation, fracture, contact forces are matter of potentials, electronic interactions, affinity, and reactivity of particles of colliding bodies in relation also to their atomic arrangement. Can we still call it only impact mechanics? Probably not. Some studies have been published about the protection capabilities of graphene nanoarmours via molecular dynamics [see Ozden et al. (2014) and Shang and Wang (2014)]: many considerations can be done with these simulations, which allow the modeling of systems up to $1 \mathrm{M}$ atoms. However, the uncertainty and insensitivity of the behavior at this 


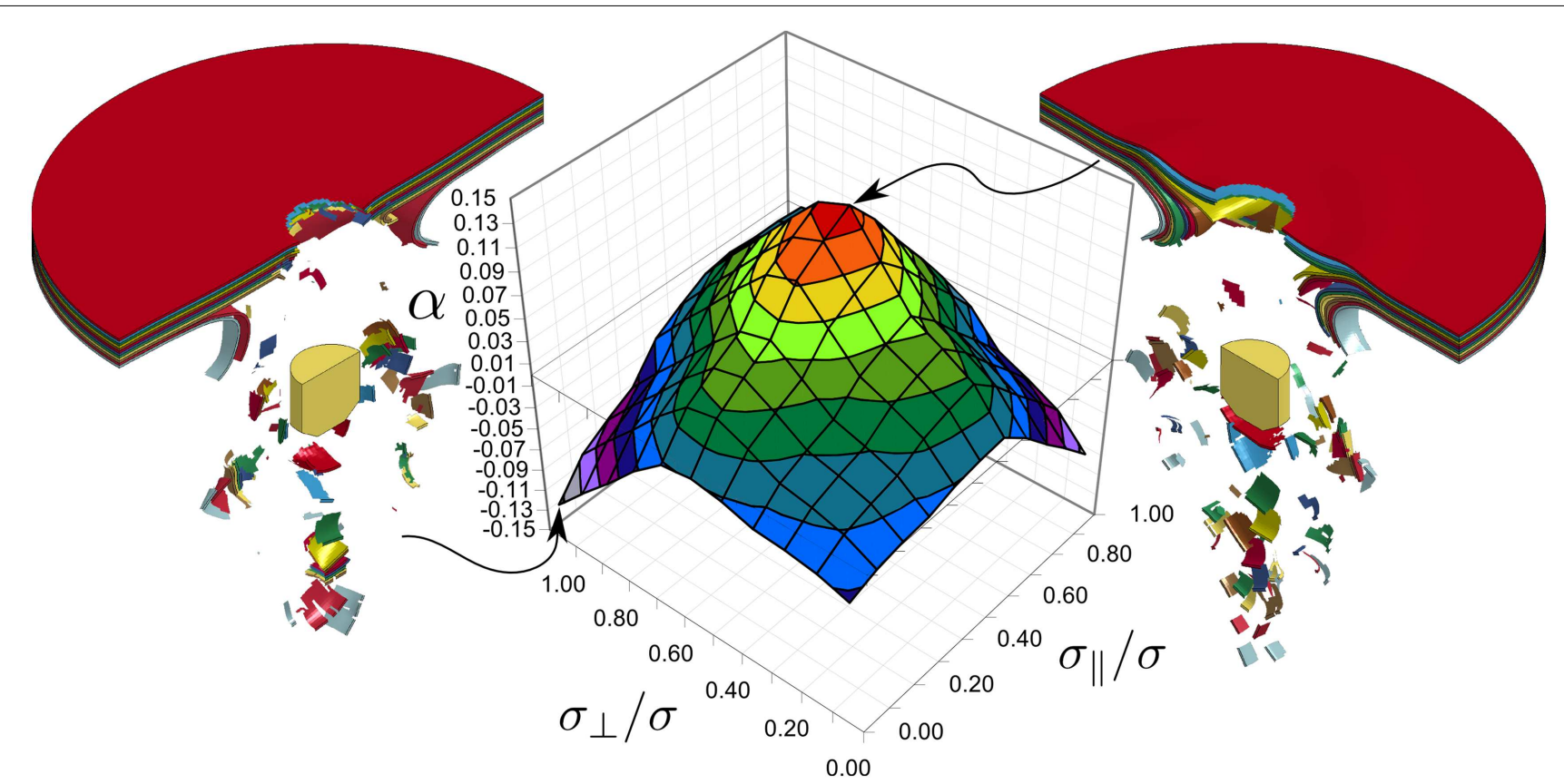

FIGURE 1 | Scalings of the absorbed energy $\boldsymbol{K}_{\text {abs }}$ in perforated multilayer panels with the number of layers $N\left(\frac{K_{\mathrm{abs}}}{N} \propto N^{\alpha}\right)$ for different values of adhesive interface normal $\left(\sigma_{\perp}\right)$ and shear $\left(\sigma_{\|}\right)$limit stresses, normalized with the plate omogenized tensile strength $\sigma$, Signetti and Pugno (2014). Results of impact FEM simulations show the existence of optimal interface parameters that increase and maximize the scaling of specific energy absorption (exponent $\alpha$ ), influencing the interaction of layers and the failure behavior. This explains controversial (positive or negative value of $\alpha$ ) experimental observations that can be found in literature (Jacobs and van Dingenen, 2001) for plates with different compaction pressure during the production process. Each point of the graph is the result of 6 configurations with different layer number $N$ overall resulting 726 simulations: the same number of cases would be extremely difficult to be performed and analyzed with ballistic experimental tests. scale would suggest the use $a b$ initio methods, inspite of their limitation to a few thousands atoms (e.g., modeling of fewlayer graphite): epitaxy simulations (Verucchi et al., 2012; Taioli et al., 2013) represent a starting knowledge base as well as a collateral application.

Moving up the mesoscale level, one of the main challenges is the modeling of multiple crack nucleation and propagation. Presently, merging more than 3 levels of hierarchy is computationally unfeasible. Some methods have been developed to overcome the problems of the FEM method (erosion mesh-sensitive approach) even if each of them shows known limitations, like the cohesive zone elements (mesh sensitiveness, remeshing required) and the extended finite element method (not applicable for multiple crack interaction). Silling (2000) has proposed a nonlocal reformulation of the standard continuum theory of solid mechanics, called peridynamics. Unlike the partial differential equations of the standard theory, the integral equations of peridynamics are applicable even when cracks and other singularities appear in the deformation field. Thus, continuous and discontinuous media can be modeled with a single set of equations. This theory naturally yields into a meshless method (Silling and Askari, 2005): this has been implemented, for instance, in the acknowledged molecular dynamics code LAMMPS (Sandia, 2015). We believe that this is a promising step toward a real multiscale approach (atomistic to continuum or within the continuum) in the same simulation.

Another advance we believe to be very interesting in the field is the isogeometric formulation (Hughes et al., 2005; Cottrell et al., 2009; Temizer et al., 2011), that offers the possibility of integrating finite element analysis with conventional Non-Uniform Rational B-Splinesbased CAD design tools. NURBS work both as geometry descriptors and element basis functions, following the same philosophy of isoparametric elements. Using NURBS it is easy to construct surfaces with $C^{1}$ or higher order of continuity and compared to $\mathrm{C}^{0}$ finite element geometry. Thus, isogeometric analysis is especially attractive to contact analysis of complex geometries (e.g., undulation, even hierarchical). This should not be limited just to impacts on complex surfaces, let us also think of modeling any problem in which adaptive and tunable wrinkling, e.g., fewlayer graphene as emblematic case (Zang et al., 2013), is exploited to provide superhydrophobicity and self-cleaning properties to surfaces. The integration with CAD would let models to be designed, tested, and adjusted on the go, with a relevant gain in time.

Being still in a relatively primordial phase in the development of these methods it is a gamble to forecast a breakthrough in simulation of bio-inspired and hierarchical nanomaterials for armors; however, it is worth keeping an eye on them since we believe them to be very promising. For sure, a synergistic combination of different and complementary research tools and multidisciplinary expertise (materials science, solid and fluid mechanics, physics) will be 
essential to lead in the next years to predictive models and optimization tools. It will be the task of simulation to support good ideas, even futuristic, pushing technology to actually switch ideas to tangible innovation for a new generation of advanced bioinspired (nano)armors with significantly improved specific penetration resistance and energy absorption capability.

\section{ACKNOWLEDGMENTS}

NMP is supported by the European Research Council (ERC StG Ideas 2011 BIHSNAM no. 279985 on "Bio-Inspired Hierarchical Super-Nanomaterials," ERC PoC 2013-I REPLICA2 no. 619448 on "Large area replication of biological antiadhesive nanosurfaces," ERC PoC 2013-II KNOTOUGH no. 632277 on "Supertough knotted fibers"), by the European Commission under the Graphene FET Flagship (WP10 "Nanocomposites," no. 604391) and by the Provincia Autonoma di Trento ("Graphene Nanocomposites," no. S116/2012-242637 and reg. delib. no. 2266). SS acknowledges support from BIHSNAM. The authors thank Ettore Barbieri for discussion and Ludovic Taxis for the English review.

\section{REFERENCES}

Abrate, S. (1998). Impact on Composite Structures. Cambridge: Cambridge University Press.

Cottrell, J. A., Hughes, T. J. R., and Bazilevs, T. (2009). Isogeometric Analysis: Toward Integration of CAD and FEA. Chichester: John Wiley \& Sons.

Cranford, S. W., Tarakanova, A., Pugno, N. M., and Buehler, M. J. (2012). Nonlinear material behaviour of spider silk yields robust webs. Nature 482, 72-78. doi:10.1038/nature10739

Cuniff, P. M. (1999). "Dimensionless parameters for optimization of textile-based body armor systems," in Proceedings of the 18th International Symposium on Ballistics, 1303-1310.

Ghosh, R., Ebrahimi, H., and Vaziri, A. (2014). Contact kinematics of biomimetic scales. Appl. Phys. Lett. 105, 233701. doi:10.1063/1.4903160

Goldsmith, W. (1999). Non-ideal projectile impact on targets. Int. J. Impact Eng. 22, 95-395. doi:10.1016/ S0734-743X(98)00031-1

Goldsmith, W. (2001). Impact - The Theory and Physics of Colliding Solids, 2nd Edn. Mineola, NY: Dover Publications.

Hoog, P. J. (2006). Composites in armour. Science 314, 1100-1101. doi:10.1126/science. 1131118

Hughes, T. J. R., Cottrer, J. A., and Bazilevs, T. (2005). Isogeometric analysis: cad, finite elements, nurbs, exact geometry and mesh refinement. Comput. Methods Appl. Mech. Eng. 194, 4135-4195. doi:10. 1016/j.cma.2004.10.008

Jacobs, M. J. N., and van Dingenen, J. L. J. (2001). Ballistic protection mechanisms in personal armour. J. Sci. Mater. 36, 3137-3142. doi:10.1023/ A: 1017922000090
Lai, C., and Ortiz, C. (2010). Potential Applications of the Natural Design of Internal Explosion Chambers in the Bombardier Beetle (Carabidae, Brachinus). Boston, MA.

Lee, C., Wei, X., Kysar, J. W., and Hone, J. (2008). Measurement of the elastic properties and intrinsic strength of monolayer graphene. Science 321, 385-388. doi:10.1126/science.1157996

Lee, J.-H., Loya, P. E., Lou, J., and Thomas, E. L. (2014). Dynamic mechanical behavior of multilayer graphene via supersonic projectile penetration. Science 346, 1092-1096. doi:10.1126/science. 1258544

Lee, J.-H., Veysse, D., Singer, J. P., Retsch, M., Saini, G., Pezeril, T., et al. (2012). High strain rate deformation of layered nanocomposites. Nat. Commun. 3, 1-7. doi:10.1038/ncomms2166

Milne, G., Deck, C., Bourdet, N., Carreira, R. P., Allinne, Q., Gallego, A., et al. (2014). Bicycle helmet modeling and validation under linear and tangential impacts. Int. J. Crashworth. 19, 323-333. doi:10.1080/13588265.2013.859470

NASA. (2015). International Space Station Risk of Impact from Orbital Debris. Available at: http:// www.nasa.gov/externalflash/iss_impact_risk/

NIST. (2005). Federal Building and Fire Safety Investigation of the World Trade Center Disaster: Final Report of the National Construction Safety Team on the Collapses of the World Trade Center Towers.

Ozden, S., Autreto, P. A., Tiwary, C. S., Khatiwada, S., Machado, L., Galvao, D. S., et al. (2014). Unzipping carbon nanotubes at high impact. Nano Lett. 14, 4131-4137. doi:10.1021/nl501753n

Patek, S. N., Korff, W. L., and Caldwell, R. L. (2005). Extreme impact and cavitation forces of a biological hammer: strike forces of the peacock mantis shrimp odontodactylus scyllarus. J. Exp. Biol. 208, 3655-3664. doi:10.1242/jeb.01831

Pugno, N. M., Coluci, V. R., and Galvao, D. S. (2007). "Nanotube- or graphene-based nanoarmors," in Computational \& Experimental Analysis of Damaged Materials, ed. D. G. Pavlou (Trivandrum: Transworld Research Network), 145-154.

Sandia National Labs. (2015). Lammps Molecular Dynamics Simulator. Available at: http://lammps. sandia.gov/

Schweizerhof, K., Nilsson, L., and Hallquist, J. O. (1992). Crashworthiness analysis for the automotive industry. Int. J. Comput. Appl Technol. 5, 134-156.

Shang, H., and Wang, W. (2014). "Shock responses of graphene reinforced composites via molecular dynamics simulations," in Proceedings of the 24th International Conference of the International Association for the Advancement of High Pressure Science and Technology and 18th International Conference of the APS Topical Group on Shock Compression of Condensed Matter (24th AIRAPT and 18th APSSCCM, Seattle, Washington, USA July 2013), 500, 112059.

Signetti, S., and Pugno, N. M. (2014). Evidence of optimal interfaces in bio-inspired ceramiccomposite panels for superior ballistic protection. J. Eur. Ceram. Soc. 34, 2823-2831. doi:10.1016/j. jeurceramsoc.2013.12.039

Silling, S. A. (2000). Reformulation of elasticity theory for discontinuities and long-range forces. $J$. Mech. Phys. Solids 48, 175-209. doi:10.1016/S00225096(99)00029-0
Silling, S. A., and Askari, E. (2005). A meshfree method based on the peridynamic model of solid mechanics. Comput. Struct. 83, 1526-1535. doi:10.1016/j. compstruc.2004.11.026

Stronge, W. J. (2000). Impact Mechanics, 2nd Edn. Cambridge: Cambridge University Press.

Taioli, S., Garberoglio, G., Simonucci, S., a Beccara, S., Aversa, L., Nardi, M., et al. (2013). Nonadiabatic ab initio molecular dynamics of supersonic beam epitaxy of silicon carbide at room temperature. J. Chem. Phys. 138, 044701. doi:10. 1063/1.4774376

Temizer, T., Wriggers, P., and Hughes, T. J. R. (2011) Contact treatment in isogeometric analysis with nurbs. Comput. Methods Appl. Mech. Eng. 200, 1100-1112. doi:10.1016/j.cma.2010.11.020

Tempelman, E., Dwaikat, M. M. S., and Spitás, C. (2012). Experimental and analytical study of freefall drop impact testing of portable products. Exp. Mech. 52, 1385-1395. doi:10.1007/s11340011-9584-y

Thielen, M., Schmitt, C. N. Z., Eckert, S., Speck, T., and Seidel, R. (2013). Structure-function relationship of the foam-like pomelo peel (citrus maxima) - an inspiration for the development of biomimetic damping materials with high energy dissipation. Bioinspir. Biomim. 8, 025001. doi:10.1088/ 1748-3182/8/2/025001

Verucchi, R., Aversa, L., Nardi, M. V., Taioli, S., a Beccara, S., Alfè, D., et al. (2012). Epitaxy of nanocrystalline silicon carbide on si(111) at room temperature. J. Am. Chem. Soc. 134, 17400-17403. doi:10.1021/ja307804v

Yang, W., Sherman, V. R., Gludovatz, B., Mackey, M., Zimmermann, E. A., Chang, E. H., et al. (2014). Protective role of arapaima gigas fish scales: structure and mechanical behavior. Acta Biomater. 5, 3599-3614. doi:10.1016/j.actbio.2014.04.009

Zang, J., Ryu, S., Pugno, N. M., Wang, Q., Tu, Q., Buehler, M. J., et al. (2013). Multifunctionality and control of the crumpling and unfolding of largearea graphene. Nat. Mater. 12, 321-325. doi:10. 1038/nmat3542

Zhang, T., Li, X., Kadkhodaei, S., and Gao, H. (2012). Flaw insensitive fracture in nanocrystalline graphene. Nano Lett. 12, 4605-4610. doi:10.1021/ nl301908b

Conflict of Interest Statement: The authors declare that the research was conducted in the absence of any commercial or financial relationships that could be construed as a potential conflict of interest.

Received: 02 February 2015; accepted: 17 February 2015; published online: 28 April 2015.

Citation: Signetti S and Pugno NM (2015) Frontiers in modeling and design of bio-inspired armors. Front. Mater. 2:17. doi: 10.3389/fmats.2015.00017

This article was submitted to Mechanics of Materials, a section of the journal Frontiers in Materials.

Copyright (c) 2015 Signetti and Pugno. This is an openaccess article distributed under the terms of the Creative Commons Attribution License (CC BY). The use, distribution or reproduction in other forums is permitted, provided the original author(s) or licensor are credited and that the original publication in this journal is cited, in accordance with accepted academic practice. No use, distribution or reproduction is permitted which does not comply with these terms. 